\title{
Higher Incidence Rates of Comorbidities in Patients with Psoriatic Arthritis Compared with the General Population Using U.S. Administrative Claims Data
}

\author{
Jeffrey Kaine, MD; Xue Song, PhD; Gilwan Kim, PharmD; \\ Peter Hur, PharmD; and Jacqueline B. Palmer, PharmD
}

\begin{abstract}
BACKGROUND: Psoriatic arthritis (PsA) is associated with multiple comorbid conditions, including cardiovascular (CV) comorbidities that impose a considerable burden on patients. Effective management of PsA requires an understanding of comorbidity profiles.
\end{abstract}

OBJECTIVE: To compare the frequency and incidence rates of comorbidities and hospitalizations among newly diagnosed PsA patients and a matched general population without PsA, using large national claims databases in the United States.

METHODS: This retrospective observational study used MarketScan databases from January 1, 2008, to September 30, 2015, to identify adult patients with newly diagnosed PsA (i.e., no PsA diagnosis during the 1 year before the first observed PsA diagnosis). The earliest date of PsA diagnosis was defined as the index date. Patients with no PsA diagnosis any time during the study period (controls) were directly matched to PsA patients with demographic characteristics. All patients had $\geq 2$ years of medical and pharmacy coverage before the index date and $\geq 1$ year of follow-up. Incident rates per 100 person-years for comorbidities of interest were evaluated. The hazard ratios of having various comorbid conditions for PsA patients were estimated by Cox proportional hazards models. All-cause and CV-related hospitalizations during the follow-up period were evaluated.

RESULTS: A total of 14,898 PsA patients and 35,037 matched controls met the study criteria. Compared with controls, PsA patients had a higher risk of $\mathrm{CV}$ disorders (incidence rate $=6.5$ vs. $5.8 ; \mathrm{HR}=1.46 ; 95 \% \mathrm{Cl}=1.37-1.56$ ) and a higher risk of the majority of the specific CV disorders (hypertension, hyperlipidemia, coronary artery disease, cerebrovascular disease, peripheral vascular disease). PsA patients also had a higher risk for any autoimmune disease (incidence rate $=8.4$ vs. 1.6 ; $H R=18.26$;

$95 \% \mathrm{Cl}=17.18-19.40$ ) and most autoimmune categories (psoriasis, ankylosing spondylitis, rheumatoid arthritis, multiple sclerosis, and other autoimmune disorders). Rates of other PsA-related comorbidities (diabetes, anxiety, fatigue, smoking, alcohol use, obesity or overweight, depression, osteoporosis, uveitis, eczema, and gout) were also significantly higher for PsA patients. The all-cause hospitalization rate was higher among PsA patients than controls $(24.9 \%$ vs. $16.2 \% ; P<0.001)$. The CV-related hospitalization rate varied depending on whether the CV condition was the primary discharge diagnosis only or was any diagnosis on the inpatient claims. The rates of coronary artery disease hospitalizations were significantly higher in PsA patients than in controls with both methods of analysis (primary diagnosis: $0.8 \%$ vs. $0.5 \% ; P<0.001$; nonprimary diagnosis: $3.2 \%$ vs. $2.2 \% ; P<0.001)$.

CONCLUSIONS: This retrospective U.S.-based claims study found that PSA patients had a high comorbidity burden. Compared with the non-PsA population, PsA patients were associated with a higher incidence of CV comorbidities, autoimmune diseases, and other PsA-related comorbidities and a higher rate of all-cause and CV-related hospitalizations. Understanding these comorbidity profiles may provide insight on the effect of comorbid conditions on disease management and health care utilization associated with PsA.

J Manag Care Spec Pharm. 2019;25(1):122-32

Copyright $\odot 2019$, Academy of Managed Care Pharmacy. All rights reserved.

\section{What is already known about this subject}

Psoriatic arthritis (PsA) has been linked to a variety of comorbidities such as metabolic disorders, autoimmune diseases, ophthalmic disease, depression, anxiety, cardiovascular (CV) conditions, and their risk factors.

Existing studies have been conducted in the United States, Canada, and Europe, with many based on small sample sizes, and a few that used claims databases to examine the incidence of comorbidities.

\section{What this study adds}

This large real-world U.S. claims-based study demonstrated that PsA patients had higher incidence rates of CV comorbidities, autoimmune diseases, diabetes, anxiety, fatigue, smoking, alcohol use, obesity or overweight, depression, osteoporosis, uveitis, eczema, and gout than patients without PsA.

This study found that PsA patients had higher rates of all-cause and CV-related hospitalizations than patients without PsA.

P soriatic arthritis (PsA) is a chronic progressive inflammatory condition that can cause irreversible damage to joints and supporting tissue and lead to a significant negative effect on the patient's quality of life. ${ }^{1-3}$ In addition to manifesting symptoms such as redness, pain, and swelling at the affected joints, PsA also exhibits disorders of the axial skeleton, nail changes, peripheral joint inflammation, enthesitis, tenosynovitis, or dactylitis. ${ }^{1,4-6}$ The prevalence of PsA is estimated to be up to $1 \%$ of the general population, ${ }^{7,8}$ and it is more prevalent among patients with psoriasis, ranging from $6 \%$ to $42 \% .^{8}$

PsA has been linked to a variety of comorbidities such as metabolic disorders (e.g., diabetes, obesity, mild liver disease); autoimmune diseases (e.g., psoriasis, rheumatoid arthritis, 
ankylosing spondylitis, Crohn's disease, ulcerative colitis, multiple sclerosis, non-PsA/psoriasis-related rheumatologic disease); ophthalmic disease; depression; anxiety; cardiovascular (CV) conditions; and their risk factors. ${ }^{9-12}$ Many autoimmune diseases are thought to share common genetic or environment risk factors, and psoriatic patients are likely to suffer from 1 or more other autoimmune diseases. ${ }^{13}$ Similarly, higher prevalence of autoimmune diseases, such as inflammatory bowel disease and possibly giant cell arteritis, has been associated with PsA. ${ }^{14}$

Multiple studies have explored the association between PsA and various comorbidities. PsA patients have an increased prevalence of diabetes, metabolic syndrome, and obesity. ${ }^{15,16}$ A systematic review showed a significant association between type 2 diabetes and PsA, and PsA patients had a higher body mass index compared with the general population. ${ }^{17}$ In addition, metabolic syndrome and insulin resistance were independently associated with the severity of PsA. ${ }^{18}$ Uveitis is the most common autoimmune ophthalmic disease observed in PsA patients. ${ }^{19}$ Autoimmune ophthalmic diseases usually precede PsA diagnosis and advance even after adequate PsA treatment. ${ }^{20}$ Depression and anxiety affected $22.2 \%$ and $36.6 \%$ of PsA patients, respectively. ${ }^{21}$ Many studies were either conducted outside the United States or were based on small sample sizes, and few used claims databases to examine the incidence of comorbidities.

The association between PsA and major CV disorders has been identified in past studies. In a systematic review of publications between 1980 and 2011, Horreau et al. (2013) showed consistent significant association between PsA and the risk of myocardial infarction and coronary artery disease, but no significant increased risk of CV mortality associated with PsA. ${ }^{22}$ A 2017 meta-analysis of published studies up to 2015 reported increased risks of CV diseases (43\%), cerebrovascular diseases (22\%), and heart failure (31\%) in PsA patients compared with the general population. ${ }^{23}$ Lin et al. (2014) confirmed a positive association between the increased prevalence of metabolic syndrome, which embodies a series of risk factors for CV conditions, and greater carotid intima-media thickness (a measure of early atherosclerotic change) in PsA patients. ${ }^{24}$

This association is important because CV mortality has been noted as the leading cause of death in PsA patients, and circulatory disease-specific mortality in PsA was found to be $33 \%$ higher than that in the general population. ${ }^{25}$ Most of these studies examined prevalence rates of $\mathrm{CV}$, and the incidence studies were mostly conducted outside the United States (in Denmark, the United Kingdom, and Sweden). Therefore, there is limited evidence examining the incidence rates of CV disorders associated with PsA in the United States.

The existing body of literature suggests considerable clinical burden associated with PsA. A U.S. retrospective claim study reported that psoriatic patients with PsA incurred higher rates of inpatient hospitalizations (odds ratio $[\mathrm{OR}]=1.6$ ), emergency room visits $(\mathrm{OR}=1.3)$, and outpatient visits $(\mathrm{OR}=62.7)$, as well as a greater number of filled prescriptions than patients without psoriasis and PsA (incidence rate ratio $=2.3 ; P<0.050$ for all). ${ }^{9}$ This suggests that recognition and treatment of comorbidities could potentially modify this significant overall burden, especially for $\mathrm{CV}$ conditions.

The intent of this large real-world U.S. claims-based study was to provide the incidence rates and the risk of developing PsA-related comorbidities following the initial diagnosis of PsA. Additionally, we evaluated the rate of health care utilization, including hospitalizations, all-cause and CV-related, among PsA patients.

\section{Methods}

\section{Data Source and Study Design}

This retrospective observational cohort study used administrative medical and pharmacy claims data from January 1, 2008, to September 30, 2015, from the Truven Health Analytics MarketScan Commercial Claims and Encounters (Commercial) and Medicare Supplemental and Coordination of Benefits (Medicare Supplemental) databases. These databases contain enrollment information, demographic information, and inpatient medical, outpatient medical, and outpatient pharmacy claims data collected from more than 150 large, self-insured U.S. employers and health plans. The study databases satisfy the conditions set forth in sections 164.514 (a)-(b)lii of the Health Insurance Portability and Accountability Act of 1996 privacy rule regarding the determination and documentation of statistically de-identified data.

\section{Study Population}

All patients with at least 1 inpatient or 2 outpatient diagnoses of PsA (International Classification of Diseases, Ninth Revision, Clinical Modification [ICD-9-CM] 696.0) from January 1, 2008, to September 30, 2015, were identified. The 2 outpatient diagnoses were required to be at least 30 days apart, but within 365 days. The index date was defined as the date of the first PsA diagnosis, and patients with a PsA diagnosis during the 12 months before the index date were excluded in order to restrict the study population to newly diagnosed PsA patients. Patients aged $<18$ years on the index date were excluded from the study. All patients were required to have continuous medical and pharmacy coverage for at least 24 months before the index date (pre-index period) and for at least 12 months following the index date (post-index period).

The PsA patients were matched with patients without any PsA diagnosis (controls) during 2008 to 2015 by calendar year, age, gender, and geographic region in a 1:1 to 1:5 ratio. The controls were assigned the same index date as that of their matched PsA counterparts. The controls were also required to have a preindex period of 24 months and a post-index period of at least 


\section{FIGURE 1 Overview of Patient Selection}

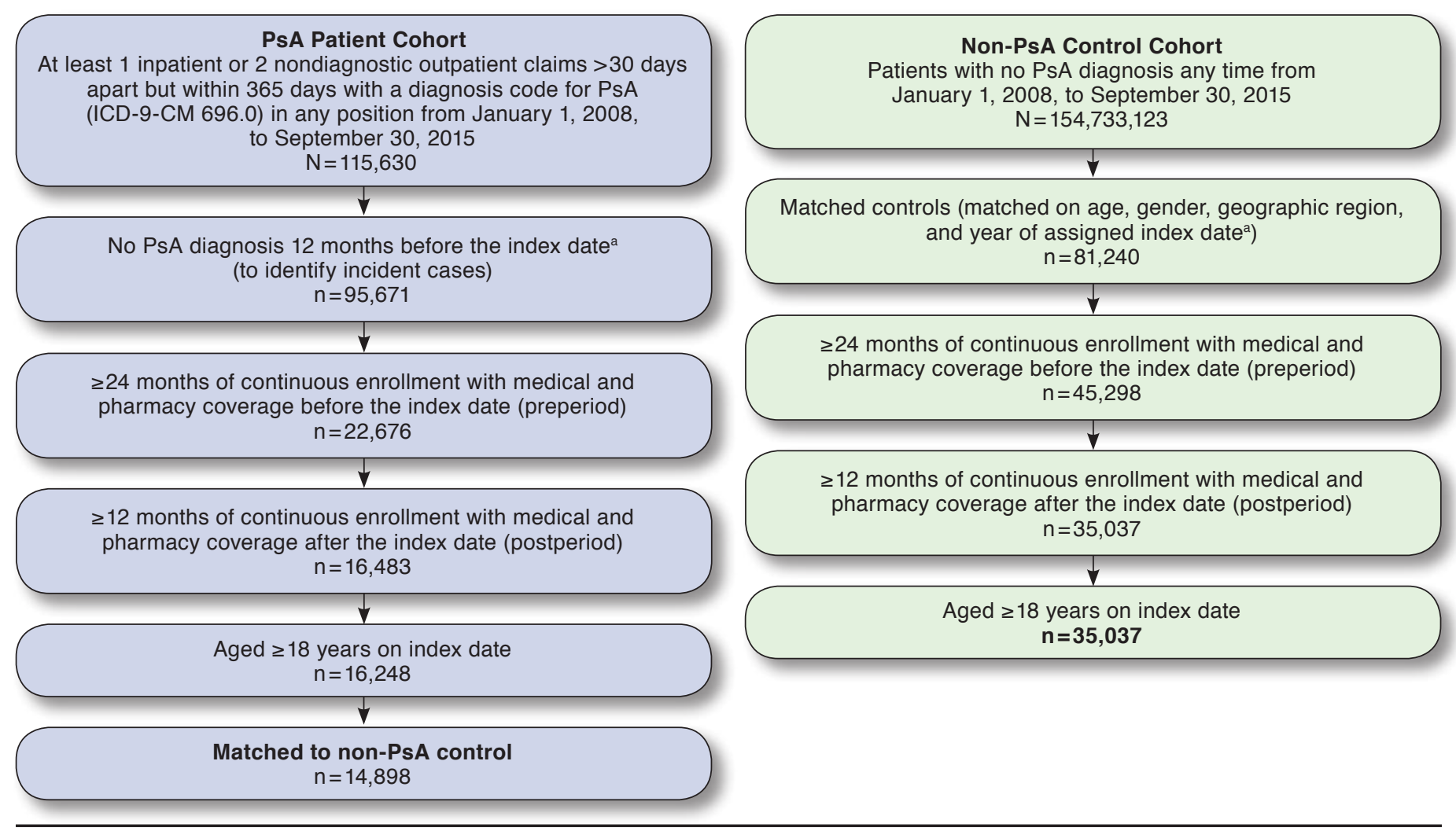

${ }^{a}$ For each patient, the index date was set to the date of the first PSA diagnosis for PsA patients or randomly assigned for non-PSA controls. ICD-9-CM = International Classification of Diseases, Ninth Revision, Clinical Modification; PsA = psoriatic arthritis.

12 months (Figure 1). The duration of follow-up was at least 12 months and spanned the index date to the earliest occurrence of inpatient death, end of continuous enrollment, or end of the study period (September 30, 2015).

\section{Baseline Demographic and Clinical Characteristics}

Demographic characteristics captured included age, gender, U.S. Census geographic region, health plan type, and urbanicity recorded on the index date. Baseline general health status was measured by the Deyo Charlson Comorbidity Index. ${ }^{26,27}$ Binary flags for all comorbidities associated with PsA during the 24-month pre-index period were reported.

\section{Study Outcomes}

The primary outcome of the study was the incidence rates of the newly diagnosed comorbidities during the follow-up period. Patients with the preexisting conditions during the 24-month pre-index period were excluded from the evaluation of incidence rate for a given comorbid condition. The incidence rate was calculated by dividing the number of patients with a newly diagnosed comorbidity by the total number of observation days. Observation days were the accumulated days from the index date until the first event date for patients diagnosed with the comorbidity and the entire follow-up period for patients without such an event. The rate was standardized as a rate per 100 person-years. Based on evidence of at least 1 diagnosis claim, the following comorbid conditions were identified and captured: CV conditions, including hypertension, hyperlipidemia, coronary artery disease, cerebrovascular disease, peripheral vascular disease, and carotid artery disease (those with at least 1 procedure for carotid endarterectomy); autoimmune diseases, such as psoriasis, ankylosing spondylitis, rheumatoid arthritis, Crohn's disease, ulcerative colitis, multiple sclerosis, and other autoimmune disorder; cancer; and other comorbidities, including diabetes (treated with or without insulin), anxiety, depression, obesity/overweight, osteoporosis, uveitis, liver disease, eczema, and gout. Asymptomatic hyperuricemia was not captured in this data analysis. Incidence rates between PsA patients and matched controls were compared.

As a secondary study outcome, all-cause and CV-related hospitalizations were reported in PsA patients and the matched controls. Two sets of CV-related hospitalizations were defined, 
Higher Incidence Rates of Comorbidities in Patients with Psoriatic Arthritis

Compared with the General Population Using U.S. Administrative Claims Data

\section{TABLE 1 Patients' Demographic and Clinical Characteristics}

\begin{tabular}{|c|c|c|c|}
\hline & $\begin{array}{c}\text { PsA Patients } \\
\mathrm{n}=14,898\end{array}$ & $\begin{array}{c}\text { Matched Controls } \\
\mathbf{n}=35,037\end{array}$ & $P$ Value \\
\hline \multicolumn{4}{|l|}{ Demographic characteristics on index date } \\
\hline Age, years, mean (SD) & $53.4(12.4)$ & $54.8(12.4)$ & $<0.001$ \\
\hline Female, $\mathrm{n}(\%)$ & $8,254 \quad(55.4)$ & $19,481 \quad(55.6)$ & 0.684 \\
\hline Urbanicity, n (\%) & $12,560 \quad(84.3)$ & $29,630 \quad(84.6)$ & 0.365 \\
\hline Geographic region, n (\%) & & & 0.001 \\
\hline Northeast & $2,838 \quad(19.0)$ & $6,302 \quad(18.0)$ & \\
\hline North Central & $3,362 \quad(22.6)$ & $8,357 \quad(23.9)$ & \\
\hline South & $5,845 \quad(39.2)$ & $13,495 \quad(38.5)$ & \\
\hline West & $2,810 \quad(18.9)$ & $6,809 \quad(19.4)$ & \\
\hline Unknown & $43 \quad(0.3)$ & $74 \quad(0.2)$ & \\
\hline Health insurance type, n (\%) & & & $<0.001$ \\
\hline Commercial & $12,639 \quad(84.8)$ & $28,695 \quad(81.9)$ & \\
\hline Medicare & $2,259 \quad(15.2)$ & $6,342 \quad(18.1)$ & \\
\hline Index year, n (\%) & & & $<0.001$ \\
\hline 2008 & $1,621 \quad(10.9)$ & $3,490 \quad(10.0)$ & \\
\hline 2009 & $1,667 \quad(11.2)$ & $3,423 \quad(9.8)$ & \\
\hline 2010 & $2,164 \quad(14.5)$ & $5,130 \quad(14.6)$ & \\
\hline 2011 & $2,609 \quad(17.5)$ & $6,335 \quad(18.1)$ & \\
\hline 2012 & $2,288 \quad(15.4)$ & $5,199 \quad(14.8)$ & \\
\hline 2013 & $2,545 \quad(17.1)$ & $6,782 \quad(19.4)$ & \\
\hline 2014 & $2,004 \quad(13.5)$ & $4,678 \quad(13.4)$ & \\
\hline Length of follow-up, years, mean (SD) & $3.0 \quad(1.7)$ & $3.0 \quad(1.6)$ & 0.445 \\
\hline \multicolumn{4}{|l|}{ Clinical characteristics during 24-month preperiod } \\
\hline Deyo Charlson Comorbidity Index, mean (SD) & $0.9 \quad(1.4)$ & $0.6 \quad(1.3)$ & $<0.001$ \\
\hline \multicolumn{4}{|l|}{ Comorbidities associated with PsA, n (\%) } \\
\hline Cardiovascular & $8,400 \quad(56.4)$ & $17,138 \quad(48.9)$ & $<0.001$ \\
\hline Hypertension & $6,297 \quad(42.3)$ & $12,379 \quad(35.3)$ & $<0.001$ \\
\hline Hyperlipidemia & $5,038 \quad(33.8)$ & $10,412 \quad(29.7)$ & $<0.001$ \\
\hline Coronary artery disease & $976 \quad(6.6)$ & $1,733 \quad(4.9)$ & $<0.001$ \\
\hline Cerebrovascular disease & $469 \quad(3.1)$ & $892 \quad(2.5)$ & $<0.001$ \\
\hline Peripheral vascular disease & $413 \quad(2.8)$ & $786 \quad(2.2)$ & $<0.001$ \\
\hline Carotid artery disease ${ }^{a}$ & $0 \quad(0.0)$ & $(0.0)$ & N/A \\
\hline Autoimmune diseases & $9,432 \quad(63.3)$ & $1,880 \quad(5.4)$ & $<0.001$ \\
\hline Psoriasis & $7,401 \quad(49.7)$ & $324 \quad(0.9)$ & $<0.001$ \\
\hline Ankylosing spondylitis & $285 \quad(1.9)$ & $(0.1)$ & $<0.001$ \\
\hline Rheumatoid arthritis & $2,143 \quad(14.4)$ & $373 \quad(1.1)$ & $<0.001$ \\
\hline Crohn's disease & $152 \quad(1.0)$ & $122 \quad(0.3)$ & $<0.001$ \\
\hline Ulcerative colitis & $159 \quad(1.1)$ & $183 \quad(0.5)$ & $<0.001$ \\
\hline Multiple sclerosis & $75 \quad(0.5)$ & $121 \quad(0.3)$ & 0.010 \\
\hline Other autoimmune disorders ${ }^{b}$ & $812 \quad(5.5)$ & $880 \quad(2.5)$ & $<0.001$ \\
\hline Other & $7,653 \quad(51.4)$ & $13,029 \quad(37.2)$ & $<0.001$ \\
\hline Cancer & $1,191 \quad(8.0)$ & $2,898 \quad(8.3)$ & 0.302 \\
\hline Diabetes $^{\mathrm{c}}$ & $2,365 \quad(15.9)$ & $4,452 \quad(12.7)$ & $<0.001$ \\
\hline With insulin use & $36 \quad(0.2)$ & $44 \quad(0.1)$ & 0.003 \\
\hline Without insulin use & $2,329 \quad(15.6)$ & $4,408 \quad(12.6)$ & $<0.001$ \\
\hline Anxiety ${ }^{d}$ & $1,374 \quad(9.2)$ & $2,173 \quad(6.2)$ & $<0.001$ \\
\hline Fatigue $^{\mathrm{d}}$ & $2,348 \quad(15.8)$ & 3,238 & $<0.001$ \\
\hline Smokingd & $826 \quad(5.5)$ & 1,399 & $<0.001$ \\
\hline Alcohol used & $839 \quad(5.6)$ & 1,370 & $<0.001$ \\
\hline Obesity or overweight ${ }^{\mathrm{d}}$ & $1,337 \quad(9.0)$ & 1,824 & $<0.001$ \\
\hline Depressiond & $1,987 \quad(13.3)$ & 2,783 & $<0.001$ \\
\hline
\end{tabular}


Higher Incidence Rates of Comorbidities in Patients with Psoriatic Arthritis

Compared with the General Population Using U.S. Administrative Claims Data

TABLE 1 Patients' Demographic and Clinical Characteristics (continued)

\begin{tabular}{|c|c|c|c|c|}
\hline & $\begin{array}{l}\text { PsA Patients } \\
\mathrm{n}=14,898\end{array}$ & \multicolumn{2}{|c|}{$\begin{array}{c}\text { Matched Controls } \\
n=35,037\end{array}$} & $P$ Value \\
\hline \multicolumn{5}{|l|}{ Other } \\
\hline Osteoporosise & $(3.8)$ & 974 & $(2.8)$ & $<0.001$ \\
\hline With fractures & $(0.3)$ & 36 & $(0.1)$ & $<0.001$ \\
\hline Without fractures & $(3.5)$ & 938 & $(2.7)$ & $<0.001$ \\
\hline Uveitis & $(1.3)$ & 153 & $(0.4)$ & $<0.001$ \\
\hline Liver disease & $(0.2)$ & 53 & $(0.2)$ & 0.209 \\
\hline Eczema & $2,457 \quad(16.5)$ & 3,173 & $(9.1)$ & $<0.001$ \\
\hline Gout & $(5.3)$ & 606 & $(1.7)$ & $<0.001$ \\
\hline \multicolumn{5}{|c|}{$\begin{array}{l}\text { aPatients were required to have at least } 1 \text { procedure for carotid endarterectomy during the post-index period. } \\
\text { bOther autoimmune disorders included alopecia areata, celiac disease, systemic sclerosis, Sjögren's syndrome, vitiligo, chronic urticaria, systemic lupus erythematosus, } \\
\text { Addison's disease, giant cell arteritis, pulmonary fibrosis, and chronic glomerulonephritis. } \\
\text { cPatients were required to have at least } 1 \text { claim for insulin during the post-index period to be flagged as having diabetes with insulin; patients with no claim for insulin } \\
\text { during the post-index period were flagged as having diabetes without insulin. } \\
\text { dConditions were underestimated in claims data due to underdiagnosis. } \\
\text { ePatients with at least } 1 \text { claim for osteoporosis diagnosis and at least } 1 \text { claim for fractures during the post-index period were flagged as having osteoporosis with fractures. } \\
\text { Patients with at least } 1 \text { claim for osteoporosis diagnosis and no claim for fractures during the post-index period were flagged as having osteoporosis without fractures. } \\
\text { PSA = psoriatic arthritis; SD = standard deviation. }\end{array}$} \\
\hline
\end{tabular}

1 requiring a CV diagnosis as the primary discharge diagnosis and 1 allowing a CV diagnosis to be in any position on the claim. The proportion of patients with at least 1 hospitalization and the mean number of hospitalizations were measured.

\section{Statistical Analysis}

Descriptive analysis was performed to compare the study outcomes between the PsA and the control cohorts. Categorical variables were presented as counts and percentages, and continuous variables were summarized by providing means and standard deviations. Differences between PsA and controls with $P<0.050$ were considered statistically significant. In order to estimate the marginal effect of PsA on the risk of developing new comorbidities, Cox proportional hazards models were used to estimate the hazards ratios (HRs) of PsA on new diagnosed comorbidities, adjusting for covariates including age, gender, region, health plan type, urbanicity, index year, Deyo Charlson Comorbidity Index, any CV condition, cancer, any autoimmune disorder, and any other comorbidities in the preperiod. HRs were considered significant if the corresponding $95 \%$ confidence interval (CI) was $>1.0$ or $<1.0$.

\section{Results}

$\overline{\text { A total of 16,248 PsA patients were included, and 14,898 were }}$ matched to 35,037 controls. Compared with controls, the PsA patients were slightly younger (53.4 vs. 54.8 years; $P<0.001$ ). Although each control was exactly the same age as its matched PsA counterpart, each PsA patient may have been matched to more than 1 control (range, 1-5), conceivably leading to a difference in mean age between the PsA and control cohorts. About $55 \%$ of the study population consisted of women, and nearly $40 \%$ of the population resided in the South, followed by
North Central regions. Both PsA patients and the matched controls had a similar mean follow-up duration of 3 years (Table 1), and all patients had at least 1 year of follow-up.

Comparison of baseline clinical characteristics demonstrated significantly higher average Deyo Charlson Comorbidity Index scores in PsA patients than in the matched controls ( 0.9 vs. 0.6; $P<0.001)$. Furthermore, PsA patients had a significantly higher prevalence of most comorbidities than did controls, including any $\mathrm{CV}$ condition $(56.4 \%$ vs. $48.9 \%$; $P<0.001)$, any autoimmune disease (63.3\% vs. $5.4 \%$; $P<0.001$ ), and all individual CV and autoimmune diseases (Table 1).

The incidence rates per 100 person-years for any CV comorbidity (6.5 vs. 5.8) and for all individual CV comorbidities were higher in the PsA cohort than in the control cohort. Similarly, the PsA patients had higher incidence rates than controls for any autoimmune disease (8.4 vs. 1.6) and for individual conditions, including psoriasis (7.9 vs. 0.3), rheumatoid arthritis (5.5 vs. 0.2), other autoimmune disorders (2.1 vs. 0.9), and ankylosing spondylitis (0.6 vs. 0.0). In addition, incidence rates for fatigue (6.7 vs. 4.1), eczema (4.9 vs. 3.8), obesity/overweight (4.8 vs. 3.0 ), depression (4.1 vs. 2.5 ), anxiety (4.0 vs. 2.8 ), smoking ( 2.5 vs. 2.0 ), cancer (2.5 vs. 2.3), diabetes (2.4 vs. 1.6), alcohol use (2.2 vs. 1.7), osteoporosis (1.7 vs. 0.9 ), gout (1.3 vs. 0.7 ), uveitis ( 0.4 vs. 0.2 ), and liver disease (0.2 vs. 0.1 ) were higher for PsA patients than for controls (Table 2, $P<0.050$ in all comparisons).

Multivariate analysis using Cox proportional hazards models indicated a consistent pattern of significantly increased risks among the PsA patients compared with controls for developing nearly all of the comorbidities of interests, with a few exceptions. Patients with PsA were 46\% more likely to be diagnosed 
Higher Incidence Rates of Comorbidities in Patients with Psoriatic Arthritis

Compared with the General Population Using U.S. Administrative Claims Data

TABLE 2 Incidence Rates per 100 Person-Years of New Comorbidities Among PsA Patients and Controls

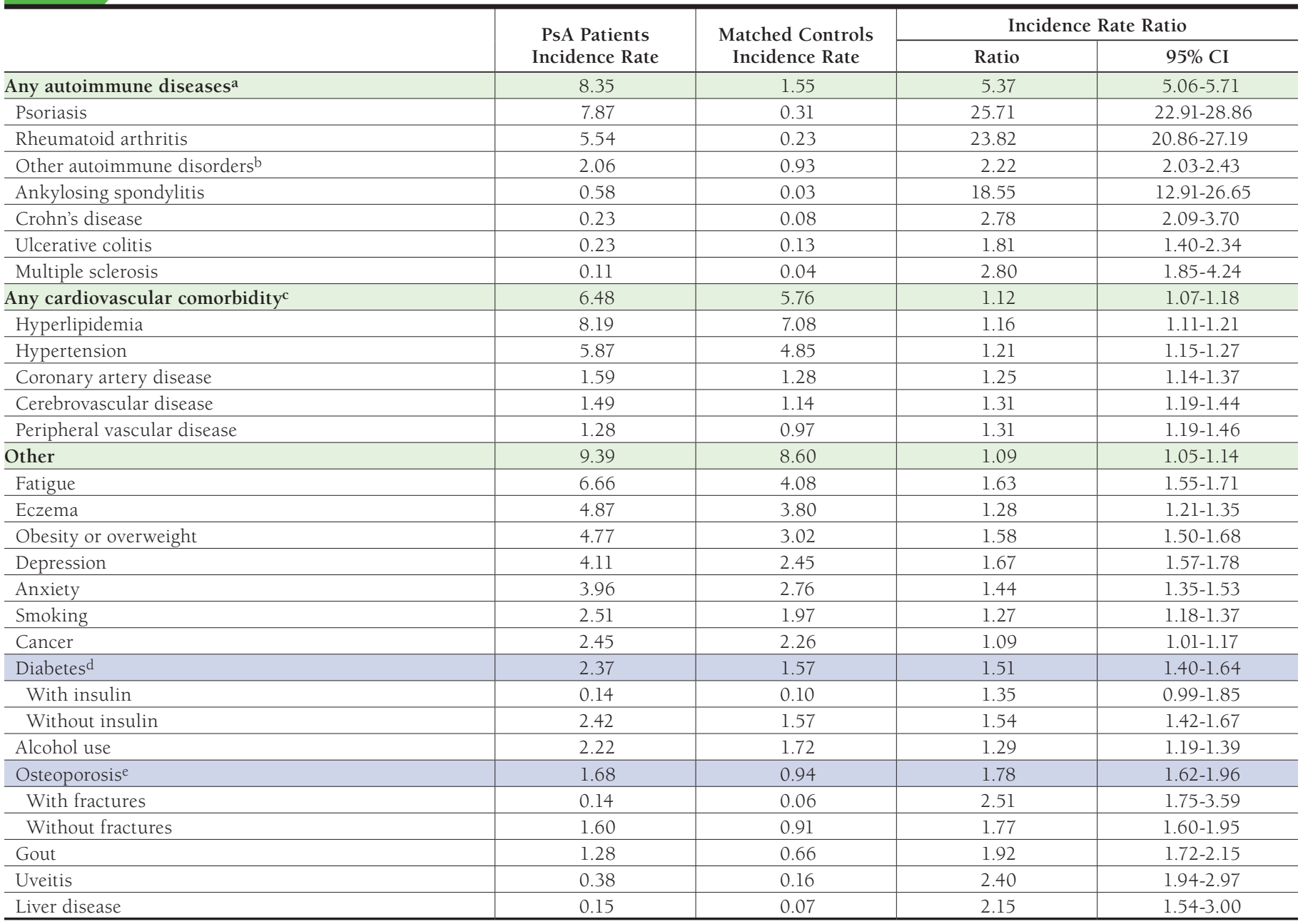

Note: $P<0.050$ in all incidence rate comparisons.

a Any autoimmune disease included psoriasis, rheumatoid arthritis, ankylosing spondylitis, Crohn's disease, ulcerative colitis, multiple sclerosis, and other autoimmune disorders.

${ }^{b}$ Other autoimmune disorders included alopecia areata, celiac disease, systemic sclerosis, Sjögren's syndrome, vitiligo, chronic urticaria, systemic lupus erythematosus, Addison's disease, giant cell arteritis, pulmonary fibrosis, and chronic glomerulonephritis.

‘Any cardiovascular comorbidities included angina, atherosclerosis, cerebrovascular disease, stroke, coronary artery disease, hypertension, myocardial infarction, peripheral vascular disease, and venous thromboembolism.

dPatients were required to have at least 1 claim for insulin during the post-index period to be flagged as having diabetes with insulin; patients with no claim for insulin during the post-index period were flagged as having diabetes without insulin.

ePatients with at least 1 claim for osteoporosis diagnosis and at least 1 claim for fractures during the post-index period were flagged as having osteoporosis with fractures.

Patients with at least 1 claim for osteoporosis diagnosis and no claim for fractures during the post-index period were flagged as having osteoporosis without fractures.

$C I=$ confidence interval; $P S A=$ psoriatic arthritis.

with any new CV condition ( $\mathrm{HR}=1.46$; 95\% CI=1.37-1.56). PsA patients also had a significantly higher risk of developing individual $\mathrm{CV}$ conditions. Likewise, compared with the controls, PsA patients were approximately 18 times more likely to have a new claim of an autoimmune disease $(\mathrm{HR}=18.26$; 95\% CI $=17.18-19.40)$, especially psoriasis ( $\mathrm{HR}=55.32 ; 95 \%$ $\mathrm{CI}=49.34-62.03)$, ankylosing spondylitis $(\mathrm{HR}=29.29 ; 95 \% \mathrm{CI}=$ 18.39-46.64), and rheumatoid arthritis ( $\mathrm{HR}=28.22 ; 95 \%$
$\mathrm{CI}=24.66-32.29)$. However, PsA was not significantly associated with a higher risk for Crohn's disease or ulcerative colitis. For other comorbidities, PsA patients had a higher risk of experiencing diabetes, anxiety, fatigue, smoking, alcohol use, obesity or overweight, depression, osteoporosis, uveitis, eczema, and gout (Figure 2). Because of the small number of patients with specific types of malignancies, the effect of PsA on various types of malignancies was not evaluated. 


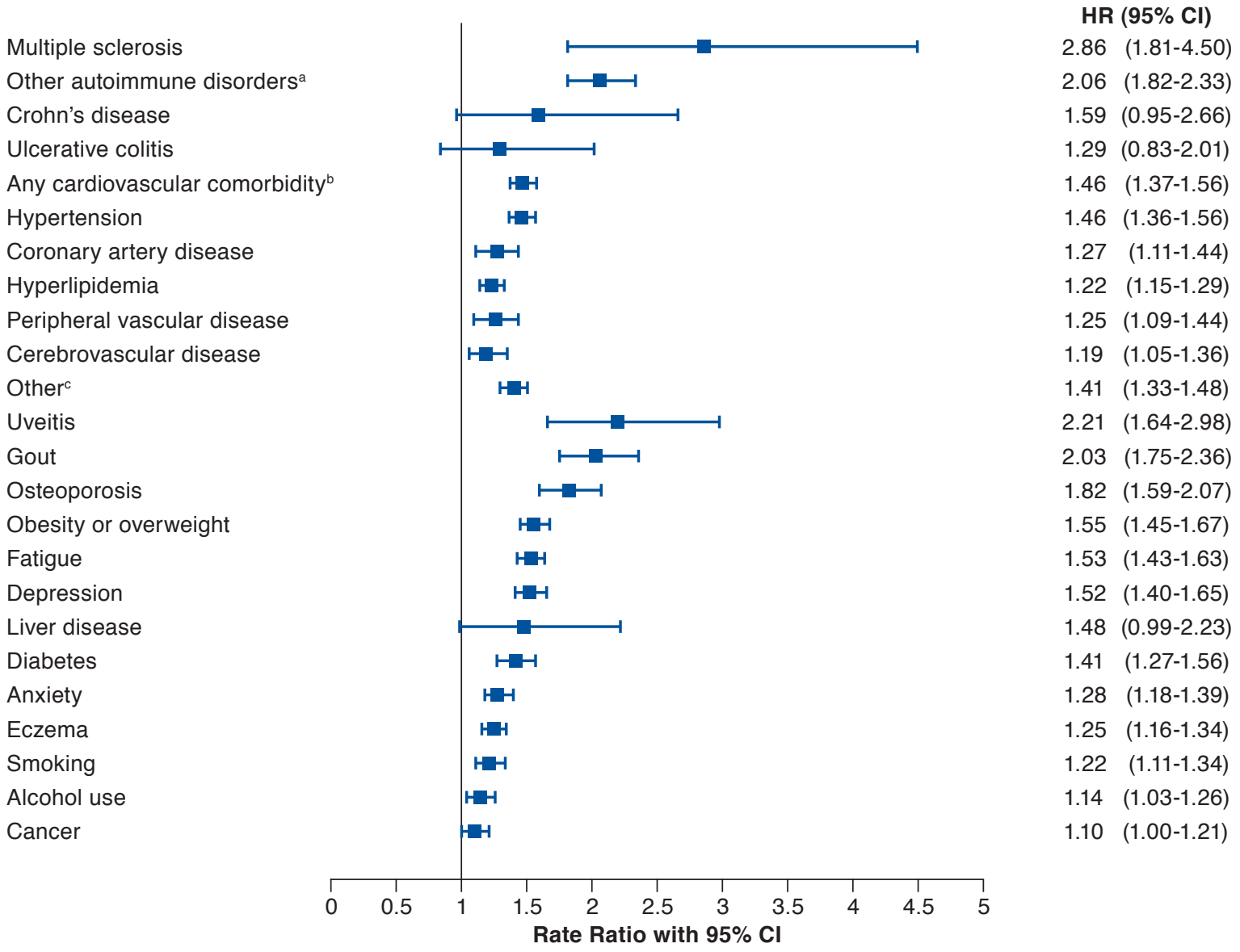

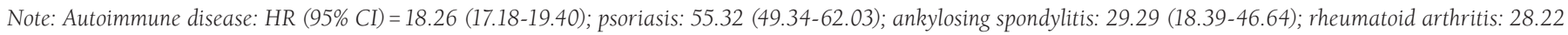
(24.66-32.29).

a Other autoimmune disorders included alopecia areata, celiac disease, systemic sclerosis, Sjögren's syndrome, vitiligo, chronic urticaria, systemic lupus erythematosus, Addison's disease, giant cell arteritis, pulmonary fibrosis, and chronic glomerulonephritis.

bAny cardiovascular comorbidity included angina, atherosclerosis, cerebrovascular disease, stroke, coronary artery disease, hypertension, myocardial infarction, peripheral vascular disease, and venous thromboembolism.

'Other included alcohol use, anxiety, cancer, depression, diabetes, eczema, fatigue, gout, liver disease, obesity or overweight, osteoporosis, smoking, and uveitis. $C I=$ confidence interval; $H R=$ hazard ratio.

Patients with PsA were significantly more likely to be hospitalized for any reason during the follow-up period compared with controls $(24.9 \%$ vs. $16.2 \% ; P<0.001)$. This difference remained significant when restricting analysis to hospitalizations with a CV diagnosis (i.e., hypertension, hyperlipidemia, coronary artery disease, cerebrovascular disease, peripheral vascular disease, or carotid artery disease) recorded in any position on an inpatient claim (14.4\% vs. 9.4\%). Hospitalization rates due to hypertension ( $11.6 \%$ vs. $7.5 \%$ ), hyperlipidemia $(5.8 \%$ vs. $3.9 \%)$, coronary artery disease (3.2\% vs. $2.2 \%)$, and cerebrovascular disease (1.7\% vs. $1.4 \%)$ were also significantly higher for PsA patients than for controls $(P<0.001$ for all except for cerebrovascular disease, in which $P=0.032$ ). Hospitalizations due to peripheral vascular disease and carotid artery disease were not significantly different between PsA patients and controls.

The mean number of all-cause hospitalizations was similar for both cohorts, but higher for PsA patients regarding any CV-specific hospitalization as well as most of the individual CV-related hospitalizations $(P<0.001$ for all except cerebrovascular disease and peripheral vascular disease). However, when limiting the analysis to hospitalizations with a CV condition as the primary discharge diagnosis, only hospitalizations specific to coronary artery disease remained 
TABLE 3 Health Care Resource Utilization During the Variable-Length Follow-up Period

Hospitalizations

PsA Patients

$\mathrm{n}=14,898$

All-cause hospitalizations

Patients with $\geq 1$ all-cause hospitalizations, $\mathrm{n}(\%)$

Number of all-cause hospitalizations, PPPM, mean (SD)

CV-related hospitalizations (with CV diagnosis as any diagnosis)

Patients with $\geq 1$ specific hospitalizations, $\mathrm{n}(\%)$

Cardiovascular

Hypertension

Hyperlipidemia

Coronary artery disease

Cerebrovascular disease

Peripheral vascular disease

Carotid artery disease

Number of specific hospitalizations, PPPM, mean (SD)

Cardiovascular

Hypertension

Hyperlipidemia

Coronary artery disease

Cerebrovascular disease

Peripheral vascular disease

Carotid artery disease

CV-related hospitalizations (with CV diagnosis as primary discharge diagnosis)

Patients with $\geq 1$ specific hospitalizations, $\mathrm{n}(\%)$

\begin{tabular}{|c|c|c|c|c|c|}
\hline Cardiovascular & 269 & (1.8) & 551 & (1.6) & 0.0609 \\
\hline Hypertension & 24 & $(0.2)$ & 87 & $(0.2)$ & 0.0583 \\
\hline Hyperlipidemia & 0 & $(0.0)$ & 0 & $(0.0)$ & N/A \\
\hline Coronary artery disease & 126 & $(0.8)$ & 186 & $(0.5)$ & $<0.001$ \\
\hline Cerebrovascular disease & 110 & $(0.7)$ & 253 & $(0.7)$ & 0.845 \\
\hline Peripheral vascular disease & 17 & $(0.1)$ & 49 & $(0.1)$ & 0.469 \\
\hline Carotid artery disease & 0 & $(0.0)$ & 0 & $(0.0)$ & N/A \\
\hline \multicolumn{6}{|c|}{ Number of specific hospitalizations, PPPM, mean (SD) } \\
\hline Cardiovascular & 0.02 & $(0.17)$ & 0.02 & $(0.16)$ & 1.000 \\
\hline Hypertension & 0.00 & $(0.04)$ & 0.00 & $(0.06)$ & 1.000 \\
\hline Hyperlipidemia & 0.00 & $(0.00)$ & 0.00 & $(0.00)$ & N/A \\
\hline Coronary artery disease & 0.01 & $(0.12)$ & 0.01 & $(0.08)$ & 1.000 \\
\hline Cerebrovascular disease & 0.01 & $(0.10)$ & 0.01 & $(0.10)$ & 1.000 \\
\hline Peripheral vascular disease & 0.00 & $(0.03)$ & 0.00 & $(0.04)$ & 1.000 \\
\hline Carotid artery disease & \multicolumn{2}{|c|}{ N/A } & \multicolumn{2}{|c|}{ N/A } & N/A \\
\hline
\end{tabular}

$C V=$ cardiovascular; $N / A=$ not available; $P P P M=$ per patient per month; $P s A=$ psoriatic arthritis; $S D=$ standard deviation

significantly higher for PsA patients than for the controls $(0.8 \%$ vs. $0.5 \% ; P<0.001 ;$ Table 3).

\section{Discussion}

This study analyzed 14,898 PsA patients and 35,037 demographically matched controls during 2008-2015 in the United States and compared incidence rates of CV conditions, autoimmune diseases, and other comorbid conditions based on the most recently available health insurance claims data. Both descriptive and multivariate results consistently suggested that PsA was associated with higher incidence rates and a higher risk of developing CV conditions, autoimmune diseases, and other PsA-related comorbidities, such as diabetes, obesity, anxiety, depression, and eczema, following the initial PsA diagnosis when compared with the non-PsA cohort.

The higher risk of CV conditions in newly diagnosed PsA patients compared with controls in this study added to the accumulating evidence of the CV comorbid burden of PsA based on studies performed in the United States and other countries. ${ }^{11,28-32}$ An increased risk of 55\% of an incident CV condition in patients with PsA compared with the general population was reported in a recent meta-analysis by Polachek et al. (2017). ${ }^{23}$ The meta-analysis was pooled from 5 significantly heterogeneous studies, including 3 from the United Kingdom, Denmark, and Sweden, and 2 U.S. studies that were abstracts only. 
Our findings showed a slightly lower $46 \%$ elevated risk of $\mathrm{CV}$ conditions, plausibly due to the requirement of at least 1 year of follow-up and the restriction of newly diagnosed PsA in this study. Unlike the CV definition used in the meta-analysis, our CV definition included hyperlipidemia and hypertension, which had the highest incidence rates (8.2 and 5.9 per 100 person-years, respectively) among the individual CV conditions examined. In a U.K. study evaluating risk of major CV conditions in 8,706 PsA patients and 82,258 randomly selected controls, significant increased risk was reported for major incident CV conditions (24\%), myocardial infarction (36\%), and stroke (33\%) in PsA patients not treated with a disease-modifying antirheumatic drug. In our study, the increased risk was 19\% for cerebrovascular disease. In this study, the HRs of PsA patients relative to controls for hypertension, hyperlipidemia, cerebrovascular disease, and peripheral vascular disease were $1.46,1.22,1.19$, and 1.25 , respectively. These findings were in line with a U.S.-based study (2001-2002, 3,066 PsA patients) reporting prevalence rates of $1.3,1.2,1.3$, and 1.6, respectively, for the same conditions. ${ }^{28}$

In our study, the PsA population showed a highly significant likelihood of developing rheumatoid arthritis $(\mathrm{HR}=28.22)$, which was higher than that of the general psoriatic patients $(\mathrm{HR}=3.6)^{13}$ but lower than for those who had moderate to severe psoriasis plus PsA (OR $=48.8) .{ }^{9}$ About $7 \%$ of PsA patients developed cancer during the follow-up period, which was similar to the non-PsA cohort. This finding corroborated previous findings of no association of PsA with cancer. ${ }^{33}$ However, for the current analysis, nonmelanoma skin cancer (NMSC) was included in this broad cancer category. If examined separately from the other cancer types, a significant correlation may exist between PsA and NMSC, as reported earlier. ${ }^{34}$ The deleterious effect of PsA on physical function, health-related quality of life, and psychological function has been well evaluated. ${ }^{35,36}$ Higher rates of fatigue and depression observed in the PsA patients in our analysis were consistent with the existing data. ${ }^{9,37}$

Considering the chronic nature of the disease and heavy burden of comorbidities, higher health care resource utilization by PsA patients is expected. This study found $24.9 \%$ of PsA patients had at least 1 all-cause hospitalization, significantly higher than the $16.2 \%$ in controls. The proportion of patients with at least 1 all-cause hospitalization was higher than the $10.8 \%$ found in patients with coexisting psoriasis and PsA in a claims-based study by Feldman et al. (2015), ${ }^{9}$ which could be attributed to the longer follow-up period in our study (average 3 years compared with 12 months in the Feldman study). The rates of hospitalizations with any $\mathrm{CV}$ diagnosis were significantly higher for the PsA patients; however, the difference became insignificant when restricting analysis to only admissions with $\mathrm{CV}$ as the primary diagnosis, possibly because the percentage of patients with CV primary hospitalizations was very low in both cohorts. Future research on PsA treatment will help provide a more complete picture of health care utilization due to comorbidities.

\section{Limitations}

Despite the strength of large population size and real-world data, this retrospective analysis has the conventional limitations of a claims-based study. First, there was the potential for misclassification of PsA and comorbid conditions as patients were identified and variables were captured through administrative claims data as opposed to medical records. Some conditions (such as anxiety, fatigue, smoking, alcohol use, overweight, obesity, and depression) are not regularly coded and their incidence rates may be underestimated. In addition, the ability to differentiate eczema from psoriasis, both clinically and histologically, can be difficult, and eczema could be miscoded as psoriasis and vice versa. Coding differences among physicians may exist and psoriasis patients seen by dermatologists may have been coded differently from patients seen by rheumatologists. The identification of newly diagnosed PsA patients was based on a 1-year clean period without PsA diagnosis, and it was possible that patients with preexisting PsA, especially those well controlled, were included in the study sample.

Second, it is possible that patients may already have had a baseline medical condition that was not treated or detected, and thus was not captured in this analysis. For example, the vast majority of PsA patients have psoriasis. This study found an incidence rate of 7.9 per 100 person-years for psoriasis in PsA patients, which could be due to the detection of a psoriatic flare rather than a new disease. In addition, physiologic mechanisms that may explain how comorbid conditions would occur after the onset of PsA are poorly understood, and the causality between comorbidities and PsA is not examined in this study.

Third, changes in accuracy or completeness of coding may have occurred during the study period (2008-2015); however, the effect of increasing coding on the PsA and the control cohorts should be similar. Future studies using the same design but based on ICD-10-CM diagnosis codes may have different results.

Fourth, there is the potential for residual confounding even after multivariate adjustment, since only characteristics that are observed in claims data can be adjusted for in the models.

Finally, this study was limited to only those individuals with at least 3 years of continuous enrollment with commercial health coverage or private Medicare supplemental coverage, and the results may not be generalizable to all PsA patients. For example, hypertension and hyperlipidemia are more likely to occur in the older population (i.e., those with Medicare supplemental coverage) than in the younger population. On the other hand, the study population came from all main geographic 
regions in the United States, and the region distribution in MarketScan is similar to that of the whole U.S. population.

The current study identified the incidence of the common comorbidities but did not examine the causality of PsA and the comorbidities. A potential explanation of the risk is that patients have some other genetic or environmental risk factor that predisposes them to both PsA and the comorbidities, especially the underlying etiology of autoimmunity. The knowledge of the frequency and risk for these diseases may help physicians anticipate and manage the conditions, as well as keeping the PsA patients informed about possible prognoses. The subsequent change in treatment due to this knowledge may lead to a reduction in health care cost and improved patient outcomes. More research in identifying the underlying cause of the common comorbidities and comprehensive economic burden is warranted to strategize the effective regimen for managing PsA.

\section{Conclusions}

This retrospective U.S.-based claims study demonstrated that PsA patients had higher incidence rates of CV comorbidities, autoimmune diseases, and other PsA-related comorbidities than non-PsA patients. PsA patients also had higher rates of all-cause and CV-related hospitalizations. Understanding these comorbidity profiles may provide insight on the effect of comorbid conditions on disease management, treatment choices, and health care burden associated with PsA.

\section{Authors}

JEFFREY KAINE, MD, Sarasota Arthritis Research Center, Sarasota, Florida. XUE SONG, PhD, and GILWAN KIM, PharmD, Truven Health Analytics, an IBM Company, Cambridge, Massachusetts. PETER HUR, PharmD, and JACQUELINE B. PALMER, PharmD, Novartis, East Hanover, New Jersey.

AUTHOR CORRESPONDENCE: Xue Song, PhD, Senior Director, Truven Health Analytics, an IBM Company, 5 Binney St., Cambridge, MA 02142. Tel.: 508.842.5312; E-mail: songx@us.ibm.com.

\section{REFERENCES}

1. National Psoriasis Foundation. Psoriatic arthritis. Available at: https:// www.psoriasis.org/sites/default/files/psoriatic_arthritis_fact_sheet_l.pdf. Accessed April 9, 2018.

2. Gelfand JM, Gladman DD, Mease PJ, et al. Epidemiology of psoriatic arthritis in the population of the United States. J Am Acad Dermatol. 2005;53(4):573

3. van de Kerkhof PC, Reich K, Kavanaugh A, et al. Physician perspectives in the management of psoriasis and psoriatic arthritis: results from the population-based Multinational Assessment of Psoriasis and Psoriatic Arthritis survey. J Eur Acad Dermatol Venereol. 2015;29(10):2002-10.

4. World Health Organization. Global report on psoriasis. 2016. Available at: http://apps.who.int/iris/bitstream/10665/204417/1/9789241565189_eng.pdf. Accessed April 2, 2018

5. Aletaha D. The many faces of psoriatic arthritis-a challenge to treatment to target? Reumatologia. 2016;54(1):1-2.

6. Liu JT, Yeh HM, Liu SY, Chen KT. Psoriatic arthritis: epidemiology, diagnosis, and treatment. World J Orthop. 2014;5(4):537-43.

7. Catanoso M, Pipitone N, Salvarani C. Epidemiology of psoriatic arthritis. Reumatismo. 2012;64(2):66-70

8. Gladman DD, Antoni C, Mease P, Clegg DO, Nash P. Psoriatic arthritis: epidemiology, clinical features, course, and outcome. Ann Rheum Dis. 2005;64 Suppl 2:iil4-17.

9. Feldman SR, Zhao Y, Shi L, Tran MH, Lu J. Economic and comorbidity burden among moderate-to-severe psoriasis patients with comorbid psoriatic arthritis. Arthritis Care Res (Hoboken). 2015;67(5):708-17.

10. Haque N, Lories RJ, de Vlam K. A5.5 Comorbidities associated with psoriatic arthritis - a cross sectional study. Ann Rheum Dis. 2015;74(Suppl 1): A48-49.

11. Ogdie A, Schwartzman S, Husni ME. Recognizing and managing comorbidities in psoriatic arthritis. Curr Opin Rheumatol. 2015;27(2):118-26.

12. Ogdie A, Yu Y, Haynes K, et al. Risk of major cardiovascular events in patients with psoriatic arthritis, psoriasis and rheumatoid arthritis: a population-based cohort study. Ann Rheum Dis. 2015;74(2):326-32.

13. Wu JJ, Nguyen TU, Poon KY, Herrinton LJ. The association of psoriasis with autoimmune diseases. J Am Acad Dermatol. 2012;67(5):924-30.

14. Makredes M, Robinson D Jr, Bala M, Kimball AB. The burden of autoimmune disease: a comparison of prevalence ratios in patients with psoriatic arthritis and psoriasis. J Am Acad Dermatol. 2009;61(3):405-10.

15. Labitigan M, Bahce-Altuntas A, Kremer JM, et al. Higher rates and clustering of abnormal lipids, obesity, and diabetes mellitus in psoriatic arthritis compared with rheumatoid arthritis. Arthritis Care Res (Hoboken). 2014;66(4):600-07.

16. Coto-Segura P, Eiris-Salvado N, Gonzalez-Lara L, et al. Psoriasis, psoriatic arthritis and type 2 diabetes mellitus: a systematic review and metaanalysis. Br J Dermatol. 2013;169(4):783-93.

17. Bhole VM, Choi HK, Burns LC, et al. Differences in body mass index among individuals with PsA, psoriasis, RA and the general population. Rheumatology (Oxford). 2012;51(3):552-56

18. Haroon M, Gallagher P, Heffernan E, FitzGerald O. High prevalence of metabolic syndrome and of insulin resistance in psoriatic arthritis is associated with the severity of underlying disease. J Rheumatol. 2014;41(7):1357-65.

19. Zeboulon N, Dougados M, Gossec L. Prevalence and characteristics of uveitis in the spondyloarthropathies: a systematic literature review. Ann Rheum Dis. 2008;67(7):955-59.

20. Smith JA, Thompson DJ, Whitcup SM, et al. A randomized, placebo-controlled, double-masked clinical trial of etanercept for the treatment of uveitis associated with juvenile idiopathic arthritis. Arthritis Rheum. 2005;53(1):18-23.

21. McDonough E, Ayearst R, Eder L, et al. Depression and anxiety in psoriatic disease: prevalence and associated factors. J Rheumatol. 2014;41(5):887-96.
The authors thank Shaswati Khan and Swathi Malla for assistance with medical writing.
This study was funded by Novartis. Kaine is a paid consultant for Novatis. Hur and Palmer are Novartis employees and stockowners. Song and Kin work for Truven Health Analytics, which received funding from Novartis to conduct this study.

\section{ACKNOWLEDGMENTS}


22. Horreau C, Pouplard C, Brenaut E, et al. Cardiovascular morbidity and mortality in psoriasis and psoriatic arthritis: a systematic literature review. J Eur Acad Dermatol Venereol. 2013;27 Supp 3:12-29.

23. Polachek A, Touma Z, Anderson M, Eder L. Risk of cardiovascular morbidity in patients with psoriatic arthritis: a meta-analysis of observational studies. Arthritis Care Res (Hoboken). 2017;69(1):67-74.

24. Lin YC, Dalal D, Churton S, et al. Relationship between metabolic syndrome and carotid intima-media thickness: cross-sectional comparison between psoriasis and psoriatic arthritis. Arthritis Care Res (Hoboken). 2014:66(1):97-103

25. Wong K, Gladman DD, Husted J, Long JA, Farewell VT. Mortality studies in psoriatic arthritis: results from a single outpatient clinic. I. Causes and risk of death. Arthritis Rheum. 1997;40(10):1868-72.

26. Chang HY, Weiner JP, Richards TM, Bleich SN, Segal JB. Validating the adapted Diabetes Complications Severity Index in claims data. Am J Manag Care. 2012;18(11):721-26.

27. Deyo RA, Cherkin DC, Ciol MA. Adapting a clinical comorbidity index for use with ICD-9-CM administrative databases. J Clin Epidemiol. 1992;45(6):613-19.

28. Han C, Robinson DW Jr, Hackett MV, Paramore LC, Fraeman KH, Bala MV. Cardiovascular disease and risk factors in patients with rheumatoid arthritis, psoriatic arthritis, and ankylosing spondylitis. J Rheumatol. 2006;33(11):2167-72.

29. Kondratiouk S, Udaltsova N, Klatsky AL. Associations of psoriatic arthritis and cardiovascular conditions in a large population. Perm J. 2008;12(4):4-8
30. Ahlehoff $\mathrm{O}$, Gislason GH, Charlot M, et al. Psoriasis is associated with clinically significant cardiovascular risk: a Danish nationwide cohort study. J Intern Med. 2011;270(2):147-57.

31. Gulati AM, Semb AG, Rollefstad S, et al. On the HUNT for cardiovascular risk factors and disease in patients with psoriatic arthritis: population-based data from the Nord-Trondelag Health Study. Ann Rheum Dis. 2016;75(5):819-24.

32. Sommer DM, Jenisch S, Suchan M, Christophers E, Weichenthal M. Increased prevalence of the metabolic syndrome in patients with moderate to severe psoriasis. Arch Dermatol Res. 2006;298(7):321-28.

33. Rohekar S, Tom BDM, Hassa A, Schentag CT, Farewell VT, Gladman DD Prevalence of malignancy in psoriatic arthritis. Arthritis Rheum. 2007:58(1):82-87.

34. Egeberg A, Thyssen JP, Gislason GH, Skov L. Skin cancer in patients with psoriasis. J Eur Acad Dermatol Venereol. 2016;30(8):1349-53.

35. Kavanaugh A, Helliwell P, Ritchlin CT. Psoriatic arthritis and burden of disease: patient perspectives from the population-based Multinational Assessment of Psoriasis and Psoriatic Arthritis (MAPP) survey. Rheumatol Ther. 2016;3(1):91-102.

36. Mease PJ, Menter MA. Quality-of-life issues in psoriasis and psoriatic arthritis: outcome measures and therapies from a dermatological perspective. J Am Acad Dermatol. 2006;54(4):685-704.

37. Husted JA, Tom BD, Schentag CT, Farewell VT, Gladman DD. Occurrence and correlates of fatigue in psoriatic arthritis. Ann Rheum Dis. 2009;68(10):1553-58. 